\title{
Multidrug-Resistant and Extensively Drug-Resistant Tuberculosis
}

\author{
Payam Tabarsi ${ }^{1, *} ;$ Davood Yadegarinia $^{2}$ \\ ${ }^{1}$ Clinical TB and Epidemiology Research Center, National Research Institute of Tuberculosis and Lung Diseases, Shahid Beheshti University of Medical Sciences, Tehran, IR Iran

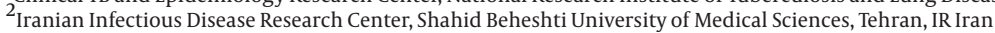 \\ ${ }^{*}$ Corresponding author: Payam Tabarsi, Clinical TB and Epidemiology Research Center, National Research Institute of Tuberculosis and Lung Diseases, Shahid Beheshti University of \\ Medical Sciences, Tehran, IR Iran. Tel/Fax: +98-2126109590, E-mail: payamtabarsi@yahoo.com
}

Received: August 4, 2014; Accepted: August 10, 2014

Multidrug-resistant (MDR) and extensively drug-resistant (XDR) tuberculosis (TB) are increasing globally. Treatment options for these patients are very limited. Although treatment of these patients with standardized regimen is associated with high mortalityand morbidity, rational usage of new drugs might be promising. In this study we will review epidemiology of XDR-TB and TDR-TB in Iran and the world.

Keywords: XDR-TB; Tuberculosis; TDR

\section{Introduction}

Drug-resistant Mycobacterium tuberculosis is a major public health problem and poses substantial obstacle to tuberculosis (TB) control. Multidrug-resistant (MDR) TB (resistant to at least isoniazid and rifampin) requires lengthy regimens of toxic drugs, which imposes high cost on the national TB program (NTP) with high mortality and morbidity (1). Extensively drug-resistant TB (XDR-TB), which is defined as TB caused by a MDR strain that is also resistant to any fluoroquinolone (FQ) and any of the second-line injectable drugs such as capreomycin, kanamycin, or amikacin, is also a new challenge in controlling TB $(2,3)$. By the end of 2012, 92 countries had reported the presence of at least one XDR-TB (1). Nevertheless, the most threatening issue is emerging of totally drug-resistant TB(TDR), which is defined as TB causes by a resistant strain to all available first-and second-line drugs (4). However, due to limitation of drug susceptibility test (DST) for second-line drugs, the practical definition for TDR is not available (5).

\section{Epidemiology}

The estimated global burden of MDR-TB in 2012 was 450000 including 300000 incidents; interestingly, about half of cases were from three countries, namely, China, India, and Russia Federation (1). Nearly 4\% of all new cases and $20 \%$ of retreatment patients were MDR-TB (1). Moreover, only one-third of the estimated cases were reported (94000 cases) (1). The majority of this gap is due to lack of standard laboratory to diagnose MDR-TB (6). By the end of 2012, 92 countries had reported cases of XDR-TB with the average prevalence of $9.6 \%$ among cases with MDR-TB. This rate is higher in some countries (21\% in Tajikistan and
$12.8 \%$ in Azerbaijan) (1). Regarding TDR, there is no formal report and all exiting data are based on reports from tertiary centers (4).

In recent years, approximately 50 cases of MDR-TB are detected annually in Iran. About half of these patients are foreigners (from Afghanistan, Azerbaijan, and Iraq) but this incident seems to be underestimation of the current status; for example, $7 \%$ of new cases and $27 \%$ of retreatment cases in 2012 had DST (1). Based on observational data that showed high rate of MDR-TB among retreatment cases, rapid escalation of DST for all retreatment cases is expected (7). Due to lack of routine DST for second-line drugs in many provinces, there is no cumulative data regarding XDR-TB. We have only some reports from tertiary centers in Tehran $(8,9)$. For better estimation of XDR-TB prevalence, routine DST for second-line drugs (quinolones and injectable agents) must be performed.

Ineffective treatment of MDR-TB due to either poor efficacy of the medications or noncompliance with longterm and more toxic therapies has led to further resistance acquisition and the emergence of XDR-TB (10). Since the XDR-TB is resistant to first-and second-line drugs, the treatment options are seriously limited. Cases with XDRTB might be virtually untreatable depending on the level of resistance to second-line drugs and the efficiency of the health system in each given setting (6). Furthermore, immunodeficiency (primary or secondary) contributes to high mortality in patients with drug-resistant $\mathrm{TB}(6,11)$.

\section{Diagnosis}

Early diagnosis of XDR-TB is a very important issue in

Copyright ( 2015, Pediartric Infections Research Center. This is an open-access article distributed under the terms of the Creative Commons Attribution-NonCommercial 4.0 International License (http://creativecommons.org/licenses/by-nc/4.0/) which permits copy and redistribute the material just in noncommercial usages, provided the original work is properly cited. 
treatment. Using solid media for diagnosing XDR-TB, the DST result only became available three to four months after initiation of anti-TB medications (12). In recent years, advances in diagnostic test has been achieved (10). The result of resistance to isoniazid and rifampin will be ready in two to three days by employing rapid genotyping tests. Hence, clinician can design a suitable treatment regimen for MDR-TB. In addition, there are rapid tests for assessing resistance to the second-line drugs that lead to early diagnosis of XDR-TB (10). Nevertheless, even in supranational reference laboratories, DST for the second-line TB drugs other than quinolones and injectable agents are very poorly standardized $(10,12)$.

\section{Treatment}

Principles of treatment of MDR-TB and XDR-TB in Iran have been reviewed in other countries (13). In a retrospective study in Iran, successful outcome was $81.2 \%$ in patients with MDR-TB in comparison with $41.6 \%$ in patients with XDR-TB (9). The mortality rate for patients with XDR-TB is about $14 \%$ to $20 \%$, which might reach to $98 \%$ in patients with HIV coinfection $(14,15)$. In another retrospective study, 15 patients with TDR-TB were reported. In that report, all the patients had failed to respond to standard second-line regimen and the results of their smear and culture remained positive after 18 months of treatment (4).

Based on these studies, it seems that TB is incurable in some cases; however, with designing the best available treatment regimen may be possible. Some new drugs had promising results (6). Linezolid is the most attractive one. In a systematic review of linezolid-containing regimen for XDR-TB, treatment success rate was $81.8 \%$ (16). In another randomized trial, early and delayed administrations of linezolid were associated with culture conversion in $70 \%$ and $35 \%$ of patients, respectively (17). Thioridazine, a neuroleptic drug, is also associated with interesting result in treatment of XDR-TB in an observational study, 15 out of 17 patients with XDR-TB had sputum conversion (18). Two new approved drugs now are on the desk: bedaquiline and delamanid. These are the first newly-released anti-TB drugs in more than 40 years (6). Other drugs with activity against XDR-TB strains are clofazimine (6), rifabutin (12), and meropenem-clavulanate (6). It seems that rational combination of these new drugs might improve treatment outcome of patients with XDR-TB and TDR-TB; however, randomized clinical trials are needed to confirm it $(6,10)$.

\section{Discussion}

XDR-TB and TDR-TB are real obstacles to controlling TB. Although treatment options are very limited, rational use of new drugs may be promising.

\section{Authors' Contributions}

Payam Tabarsi: idea and writing manuscript; Davood Yadegarinia: reviewing manuscript.

\section{References}

1. World Health Organization. Global tuberculosis report 2013. Geneva; 2013.

2. World Health Organization. Definitions and reporting framework for tuberculosis-2013 revision. Geneva; 2013.

3. Centers for Disease C, Prevention. Emergence of Mycobacterium tuberculosis with extensive resistance to second-line drugs--worldwide, 2000-2004. MMWR Morb Mortal Wkly Rep. 2006;55(11):301-5.

4. Velayati AA, Masjedi MR, Farnia P, Tabarsi P, Ghanavi J, Ziazarifi $\mathrm{AH}$, et al. Emergence of new forms of totally drug-resistant tuberculosis bacilli: super extensively drug-resistant tuberculosis or totally drug-resistant strains in iran. Chest. 2009;136(2):420-5.

5. Cegielski P, Nunn P, Kurbatova EV, Weyer K, Dalton TL, Wares DF, et al. Challenges and controversies in defining totally drug-resistant tuberculosis. Emerg Infect Dis. 2012;18(11).

6. Matteelli A, Roggi A, Carvalho AC. Extensively drug-resistant tuberculosis: epidemiology and management. Clin Epidemiol. 2014;6:111-8.

7. Tabarsi P, Nooraki A, Mirsaeidi M, Amiri M, Baghaei P, Farnia P, et al. Representative drug susceptibility patterns for guiding design of re-treatment regimens for multidrug-resistant tuberculosis in Iran. Respirology. 2008;13(1):108-11.

8. Masjedi MR, Tabarsi P, Baghaei P, Jalali S, Farnia P, Chitsaz E, et al. Extensively drug-resistant tuberculosis treatment outcome in Iran: a case series of seven patients. Int J Infect Dis. 2010;14(5):e399-402.

9. Tabarsi P, Chitsaz E, Baghaei P, Shamaei M, Farnia P, Marjani M et al. Impact of extensively drug-resistant tuberculosis on treatment outcome of multidrug-resistant tuberculosis patients with standardized regimen: report from Iran. Microb Drug Resist. 2010;16(1):81-6.

10. Tabarsi P, Mardani M. Extensively Drug-Resistant Tuberculosis: A Review Article. Arch clin infect dis. 2012;7(3):81-4.

11. Tabarsi P, Marjani M, Mansouri N, Farnia P, Boisson-Dupuis S, Bustamante J, et al. Lethal tuberculosis in a previously healthy adult with IL-12 receptor deficiency.J Clin Immunol. 2011;31(4):537-9.

12. Shim TS, Jo KW. Medical treatment of pulmonary multidrug-resistant tuberculosis. Infect Chemother. 2013;45(4):367-74.

13. Masjedi MR, Tabarsi P, Marjani M, Shiva PB, Nasehi M, Gooya MM, et al. Management of MDR-TB: Review of Iran's Experience. Tanaffos. 2013;12(1):6-15.

14. Kim HR, Hwang SS, Kim HJ, Lee SM, Yoo CG, Kim YW, et al. Impact of extensive drug resistance on treatment outcomes in non-HIVinfected patients with multidrug-resistant tuberculosis. Clin Infect Dis. 2007; 45(10):1290-5

15. Gandhi NR, Moll A, Sturm AW, Pawinski R, Govender T, Lalloo U, et al. Extensively drug-resistant tuberculosis as a cause of death in patients co-infected with tuberculosis and HIV in a rural area of South Africa. Lancet. 2006;368(9547):1575-80.

16. Sotgiu G, Centis R, D'Ambrosio L, Alffenaar JW, Anger HA, Caminero JA, et al. Efficacy, safety and tolerability of linezolid containing regimens in treating MDR-TB and XDR-TB: systematic review and meta-analysis. Eur Respir J. 2012;40(6):1430-42.

17. Lee M, Lee J, Carroll MW, Choi H, Min S, Song T, et al. Linezolid for treatment of chronic extensively drug-resistant tuberculosis. $N$ Engl J Med. 2012;367(16):1508-18.

18. Abbate E, Vescovo M, Natiello M, Cufre M, Garcia A, Gonzalez Montaner P, et al. Successful alternative treatment of extensively drug-resistant tuberculosis in Argentina with a combination of linezolid, moxifloxacin and thioridazine. JAntimicrob Chemother. 2012;67(2):473-7. 